\section{Atypical presentation of congenital pneumonia: Value of lung ultrasound}

\author{
Virginie Meau-Petit* and Grenville F Fox
}

Evelina London Neonatal Unit, Guy's and St.Thomas Hospital, London, UK

A term neonate was transferred from a Local Neonatal Unit to our surgical Neonatal Intensive Care Unit on Day 2 due to abdominal distension with radiological appearances suggestive of intestinal obstruction. He was born by Caesarean section with no risk factors for sepsis. He was intubated at birth for increased work of breathing and failed planned extubation on Day 1. Ventilation requirements were minimal and chest x-ray showed mild diffuse alveolar syndrome (Figure 1). Lung ultrasound on admission identified bilateral focal posterior lung consolidation, predominantly on the left side, surrounded with white lungs and with significant pleural effusion (Figure 2, Supplemental Video), highly suggestive of congenital pneumonia. C-reactive protein (CRP) reached a maximum of $15 \mathrm{mg} / \mathrm{l}$. After 72 hours of antibiotics, he was successfully extubated with no respiratory support. Abdominal symptoms spontaneously resolved. An upper gastro-intestinal contrast study and a rectal biopsy were normal. Lung ultrasound performed after 7 days of antibiotics showed total resolution of the pleural effusion and consolidation.

In this clinical presentation of acute abdominal distension and unexplained respiratory distress, lung ultrasound facilitated the diagnosis of congenital pneumonia with pleural effusion, which was missed by chest radiography.

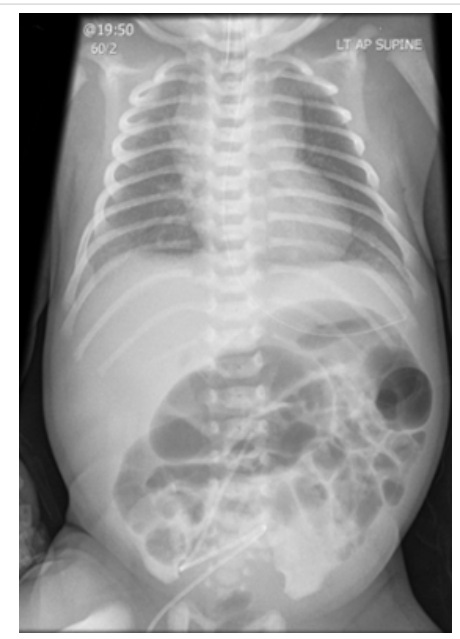

Figure 1: Chest and abdominal X-ray showing mild RDS and dilated intestinal loops.
More Information

*Address for Correspondence: Virginie Meau-Petit, Neonatal Intensive Care Unit, Evelina London Children's Hospital, Westminster Bridge Rd, London SE1 7EH, UK, Tel: + 447850286 581; Fax: + 4402071884029; Email: virginie.meau-petit@gstt.nhs.uk

Submitted: March 09, 2021

Approved: March 26, 2021

Published: March 29, 2021

How to cite this article: Meau-Petit V, Fox GF. Atypical presentation of congenital pneumonia: Value of lung ultrasound. J Adv Pediatr Child Health. 2021; 4: 033-034.

DOI: 10.29328/journal.japch.1001027

Copyright: @ 2021 Meau-Petit V, et al. This is an open access article distributed under the Creative Commons Attribution License, which permits unrestricted use, distribution, and reproduction in any medium, provided the original work is properly cited.

Keywords: Pneumonia; Ultrasound; Neonate; Intestinal pseudo-obstruction

\section{(W) Check for updates \\ OPEN ACCESS}

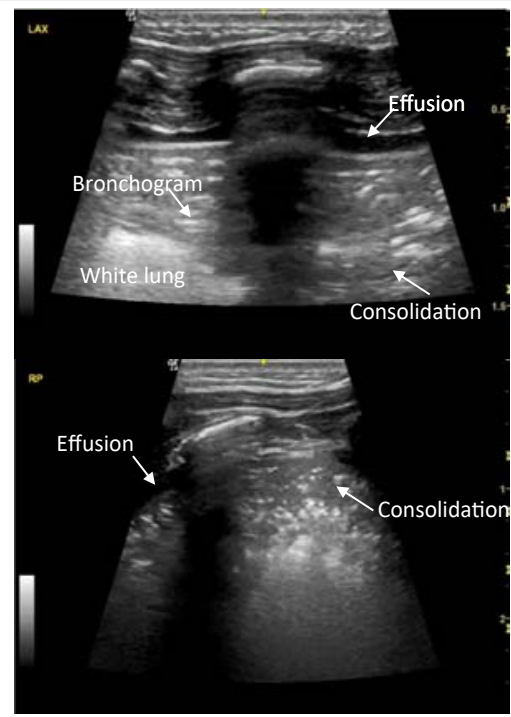

Figure 2: Lung ultrasound images: left posterior axillary (a) and right posterior (b) views, showing small effusion facing large consolidation with bronchogram and irregular borders.

Lung ultrasound is a rapid, non-irradiating, cheap, efficient and permanently available tool with a steep practitioner learning curve [1] and high inter-observer agreement [2]. In published international guidelines, congenital pneumonia was defined as the presence of consolidation with irregular borders and air bronchograms, associated with pleural line 
abnormalities, and alveolar-interstitial pattern in the adjacent areas, which is totally consistent with our findings [3]. Previous international expert consensus recommendations [4] supported the use of lung ultrasound for the diagnosis of pneumonia in neonates and children. There is currently no gold standard for the diagnosis of congenital pneumonia and diagnosis relies mainly on clinical, biological and radiology parameters. Adding lung ultrasound to the investigation of a newborn with suspected congenital pneumonia may increase the specificity of the diagnosis and should be explored further. Performing lung ultrasound in neonates with atypical clinical presentation involving respiratory symptoms could also enhance diagnosis, as suggested in our case report. There is currently no data about the different lung ultrasound patterns among various types of pneumonia (congenital, community acquired or ventilator associated), and this needs to be investigated further. Meta-analysis of lung ultrasound for the diagnosis of community acquired pneumonia in children reported a pooled sensitivity and specificity for pneumonia diagnosis of $96 \%$ and 93\%, respectively, and an area under the receiver operating curve of 0.98 , which was superior to the accuracy of chest radiography [5]. In ventilator associated pneumonia, a recent observational study performed in preterm neonates has shown an increased specificity and area under the curve when lung ultrasound is included in diagnosis criteria, compared to chest radiography [6]. Another study demonstrated poor agreement between interpreters of chest radiography to diagnose ventilator associated pneumonia in neonates [7]. In addition, recent paediatric ventilator associated event definition and the new CDC definition for ventilator associated pneumonia have removed chest radiography in their diagnosis criteria in view of the subjectivity of interpretation, lack of sensitivity and specificity [18]. Utilisation of lung ultrasound to diagnose pneumonia in intensive care has also shown to reduce cost and radiation exposure [9].

We suggest that in neonates, lung ultrasound may perform better than chest radiography, for immediate and accurate diagnosis of congenital pneumonia, especially in complex cases when respiratory symptoms are combined with other organ involvement. Further studies comparing the performance of lung ultrasound and chest radiography in congenital pneumonia are warranted.

\section{References}

1. See KC, Ong V, Wong SH, Leanda R, Santos J, et al. Lung ultrasound training: curriculum implementation and learning trajectory among respiratory therapists. Intensive Care Med. 2016; 42: 63-71.

PubMed: https://pubmed.ncbi.nlm.nih.gov/26474994/

2. Brusa G, Savoia M, Vergine M, Bon A, Copetti R, et al. Neonatal Lung Sonography: Interobserver Agreement Between Physician Interpreters With Varying Levels of Experience. J Ultrasound Med. 2015; 34: 1549-1554.

PubMed: https://pubmed.ncbi.nlm.nih.gov/26254148/

3. Raimondi F, Yousef N, Migliaro F, Capasso L, De Luca D. Point-ofcare lung ultrasound in neonatology: classification into descriptive and functional applications. Pediatr Res. 2018; 1-8.

PubMed: https://pubmed.ncbi.nlm.nih.gov/30127522/

4. Volpicelli G, Elbarbary M, Blaivas M, Lichtenstein DA, Mathis G, et al. International evidence-based recommendations for point-of-care lung ultrasound. Intensive Care Med. Springer-Verlag. 2012; 38: 577-591. PubMed: https://pubmed.ncbi.nlm.nih.gov/22392031/

5. Pereda MA, Chavez MA, Hooper-Miele CC, Robert H Gilman 3, Mark C Steinhoff et al. Lung ultrasound for the diagnosis of pneumonia in children: a meta-analysis. Pediatrics. 2015; 135: 714-722. PubMed: https://pubmed.ncbi.nlm.nih.gov/25780071/

6. Tusor N, De Cunto A, Basma Y, Klein JL, Meau-Petit V. Ventilatorassociated pneumonia in neonates: the role of point of care lung ultrasound. Eur J Pediatr. 2021;180: 137-146.

PubMed: https://pubmed.ncbi.nlm.nih.gov/32592026/

7. Goerens A, LehnickD,BüttcherM, DaetwylerK, FontanaM, etal. Neonatal Ventilator Associated Pneumonia: A Quality Improvement Initiative Focusing on Antimicrobial Stewardship. Front Pediatr. 2018; 6: 262. PubMed: https://pubmed.ncbi.nlm.nih.gov/30320046/

8. Peña-López $Y$, Pujol M, Campins M, Lagunes L, Balcells $\mathrm{J}$, et al. Assessing prediction accuracy for outcomes of ventilator-associated events and infections in critically ill children: a prospective cohort study. Clin Microbiol Infect. 2018; 24: 732-737.

PubMed: https://pubmed.ncbi.nlm.nih.gov/29031787/

9. Escourrou G, De Luca D. Lung ultrasound decreased radiation exposure in preterm infants in a neonatal intensive care unit. Acta Paediatr. 2016; $105:$ 237-239.

PubMed: https://pubmed.ncbi.nlm.nih.gov/26880491/ 